\title{
REVISIONES SISTEMÁTICAS DE LA LITERATURA: UNA NUEVA ETAPA DE LA INVESTIGACIÓN APLICADA EN LA FUCS
}

\author{
Guillermo Sánchez V. MD PhD*
}

Las revisiones sistemáticas de la literatura son un tipo específico de diseño de investigación clínica y epidemiológica que ha tomado fuerza en el mundo durante las últimas tres décadas, probablemente producto del advenimiento y fortalecimiento de la denominada corriente de medicina basada en evidencia MBE. Una revisión sistemática es un tipo de diseño especial queno parte de unidades de análisis convencional -pacientes o individuos-sino que emplea productos de investigación primaria y los integra en una síntesis cualitativa y cuantitativa (meta-análisis), cuando esto último es posible. Como toda investigación, debe partir de una pregunta concreta, que deberá ser traducida en un objetivo, el cual será desarrollado a través de métodos específicos que le confieren su carácter de sistematicidad y rigurosidad científica.

En el mundo, el propósito fundamental de las revisiones se ha centrado en evaluar la efectividad, eficacia y seguridad de alternativas terapéuticas, es por esto que el diseño idóneo se centra sobre las revisiones sistemáticas de ensayos clínicos; no obstante ahora se discute acerca del alcance, limitaciones y bondades de las revisiones sistemáticas de estudios observacionales.

La Fundación Universitaria de Ciencias de la Salud, gracias a su trayectoria en la rama de la educación en salud, posee una serie de características que la hacen una entidad de altísimo potencial para el desarrollo serio y riguroso de este tipo de trabajos de investigación. Por esta razón decidimos entrar en contacto con la Colaboración Cochrane, que es la organización internacional de mayor reconocimiento en el campo de las revisiones sistemáticas, con el objetivo de vin-

Director División de Investigaciones, Fundación Universitaria de Ciencias de la Salud.Bogotá DC, Colombia. divisioninvestigaciones@fucsalud.edu.co cularnos a esta red de trabajo y así generar productos de alto nivel de impacto y reconocimiento.

Esta intención se materializó en el año 2012, cuando en el marco de la misión académica a España tuvimos oportunidad de reunirnos en el Hospital Sant Pau de Barcelona con el doctor Xavier Bonfill, Director del Centro Cochrane Iberoamericano. En esta sesión se planteó el deseo de hacer parte de la organización y se detallaron los requisitos y compromisos que debíamos cumplir y asumir para hacerparte de la red. Volvimos a Colombia con la convicción de que sería posible lograr este objetivo; estructuramos el proyecto, vinculamos al equipo a la doctora Ingrid Arévalo, organizamos el primer curso de revisiones sistemáticas de la literatura dictado por el experto investigador Arturo Martí y por último el proyecto culminó con la firma del convenio de cooperación y la designación de la FUCS como el cuarto centro colaborador Cochrane en Colombia.

El camino no termina aquí, de hecho estamos ante la puerta de un largo recorrido en el desarrollo y consolidación de una organización capaz de generar revisiones sistemáticas de alta calidad metodológica y pertinencia científica en cada una de las especialidades de nuestro portafolio. Este aprendizaje podrá ser transferido a las otras facultades de salud de la institución y dado el potencial existente, auguro que pronto seremos un centro bandera en diferentes áreas de trabajo y lograremos un alto reconocimiento en el concierto internacional.

Felicitamos a la FUCS por un logro más en este arduo camino hacia la construcción y consolidación de una organización educativa comprometida con la generación de conocimiento científico de al to impacto social. 